\title{
La accesibilidad universal en el campus de la Universidad Nacional Autónoma de Honduras - Plan Maestro de accesibilidad - Honduras 2014
}

\author{
Luis Estrada, Gerardo Salas, Sergio Ortega ${ }^{1}$ \\ Flora Girón ${ }^{2}$
}

\section{RESUMEN}

El presente artículo ofrece el diagnóstico y los proyectos para alcanzar la accesibilidad universal en los edificios que forman parte de Ciudad Universitaria CU, según el Decreto 160 - 2005 (La Gaceta, 2005) y las Normas de Actualización de la Zonificación y Normas de Fraccionamiento, Obras y Uso del Suelo en el Distrito Central (Metroplan, 2008) además de la propuesta de una Plaza Central en el campus de la mayor universidad del país. El problema de la accesibilidad universal es un tema cualitativo, sin embargo, se realizaron encuestas para saber la percepción del estudiante universitario respecto a la accesibilidad. Existe una población discapacitada de 348 personas matriculadas en el segundo periodo de 2014. Se llevó a cabo un diagnóstico de los 33 edificios que componen el campus, las 70 rampas de circulación, los 28 estacionamientos y los 9 cruces peatonales. Posteriormente, se proyectó todas y cada una de las soluciones para volver el área accesible. Se hizo la propuesta de un estacionamiento central con capacidad para 600 vehículos y se aprovechó para proyectar una gran plaza de entrada. Los resultados indican que solamente un tercio de los edificios son accesibles, de los 17 estacionamientos pavimentados, ninguno posee plazas para discapacitados y se verificó que de las 70 rampas de circulación entre los edificios, solamente 31 tienen menos del 10\% de inclinación. En este texto, compartimos los resultados de estos aspectos como una forma de contribución con las autoridades de la UNAH, de manera que, de acuerdo a sus prioridades y programación puedan realizar estas mejoras que vendrán a beneficiar a la población estudiantil.

\footnotetext{
${ }^{1}$ Beneficiarios de una beca sustantiva, Profesores, Escuela de Arquitectura y Diseño, Facultad de Humanidades y Arte, UNAH: Correo electrónico: luis.estrada@unah.edu.hn, arquitecsalas@ yahoo.com, sortega68@yahoo.com.mx

${ }^{2}$ Beneficiarios de una beca sustantiva, Universidad Nacional Autónoma de Honduras. Vicerrectoría de Orientación y Asuntos Estudiantiles, VOAE / UNAH. Directora del Programa de Servicios a Estudiantes con Necesidades Especiales PROSENE. Correo electrónico: famgila@yahoo.com
} 
Palabras claves: accesibilidad universal, Ciudad Universitaria, rampas, estacionamientos, discapacidad, barreras arquitectónicas.

\section{ABSTRACT}

This article provides a review of accessibility in buildings belonging to Ciudad Universitaria, handicapped spaces, main parking lots and a proposal for a Central Plaza on the campus of the largest university in the country. The research has a mixed approach. The problem of universal accessibility is qualitative; however, surveys were conducted to know the perception of students regarding accessibility. A population of 348 disabled persons registered in the second quarter of 2014 was recorded. A diagnosis of all 34 buildings that make up the campus, 28 parking spaces, 9 crosswalks and all ramps was made. Subsequently, all and each of the solutions to make the area accessible were projected. The proposal suggested a central parking lot for 600 vehicles and was used to project a large entrance plaza. In this paper, we share the results of this research as a form of contribution with the authorities of the UNAH, so that according to their priorities and programming they can carry out the improvements that will come to benefit the student population.

Keywords: universal accessibility, Ciudad Universitaria, ramps, parking lots, disability, architectural barriers. 


\section{INTRODUCCIÓN}

La accesibilidad universal en un lugar donde circula constantemente una gran cantidad de personas, resulta ser un problema complejo, en el cual convergen aspectos tanto económicos, como sociales y arquitectónicos. En términos de eliminación de estas barreras, en cuanto a señalización, plazas de estacionamientos o textura de la circulación, pueden llegar a ser más sencillas o económicas de lo que se piensa.

La población con algún tipo de discapacidad es sólo la punta del iceberg del beneficio generado por la accesibilidad. Debe tomarse en cuenta que hoy en día somos considerados un país joven, es decir, que alrededor de $2 / 3$ de la población tiene menos de 30 años (INE, 2013 Honduras. XVII Censo de Población y VI de Vivienda), sin embargo, esta realidad es cambiante y el envejecimiento de la población revelará una mayor necesidad de entornos adaptados y espacios proyectados para todas las personas, independiente de sus capacidades físicas.

Los esfuerzos para mejorar accesibilidad son una inversión en capital humano y en infraestructura, consecuentemente las obras de accesibilidad revalorizan el patrimonio arquitectónico, el beneficio social de estas obras es mayor que su costo, considerando las mejoras sociales intangibles generadas por estas mejoras.

Después de las primeras construcciones en 1965, la Ciudad Universitaria emerge física y estructuralmente como una obra amplia y moderna, en un área de 120.31 manzanas. Su visión y trayectoria la perfilan como una institución líder y protagonista principal en la transformación de la sociedad hondureña mediante la formación con recursos humanos del más alto nivel académico, científico y ético.

El trabajo de investigación a que se refiere este artículo, contiene información importante en el campo de la accesibilidad relacionada a nuestro contexto. Abarca una compilación de ideas y propuestas sobre la vida cotidiana, con fuerte basamento científico y teórico, proveniente de diversos manuales y de la propia experiencia, que se pretende constituir en una herramienta para intervenir el espacio físico, con el objetivo de mejorar de la calidad de vida para hacer frente a la discapacidad en condiciones de dignidad y competencia. Se trata de eliminar o superar, por no decir derribar, las barreras arquitectónicas que se encuentran en el campus.

Se ha avanzado bastante en el tema pero aún quedan batallas importantes que ganar hasta alcanzar la accesibilidad total del espacio construido. El trabajo de investiga- 
ción se encuentra estructurado en torno a tres bloques temáticos: 1) Diagnóstico de la infraestructura arquitectónica. 2) Encuestas a la población estudiantil sobre la percepción de la accesibilidad en CU. 3) Plan de Accesibilidad Universal para el campus, que reúne todos los proyectos para tornar el espacio accesible, desde la rampa más complicada hasta la sencilla ubicación de las plazas de estacionamientos para discapacitados.

Dentro del primer bloque se encuentra la situación actual de los 33 edificios que componen el campus, los estacionamientos, las rampas y los cruces peatonales. Todos los aspectos arquitectónicos que caracterizan el campus. En el segundo bloque, se toma la percepción de accesibilidad en la población estudiantil a través de encuestas, tomando en consideración los usuarios directamente afectados. En el tercer bloque, se presentan las propuestas para solventar los problemas denunciados. Se destaca la importancia de la autonomía e independencia de las personas con alguna discapacidad.

En suma, el trabajo recoge la realidad de la accesibilidad en el espacio universitario, donde es tal el volumen de retos por abordar que nos hace ver la realidad de la discapacidad con otra perspectiva, con un enfoque sobre las necesidades educativas especiales que hay que retomar. En este sentido, se pretende cambiar el paradigma de la discapacidad como problema personal para convertirse en un problema social, educativo, económico y arquitectónico.

\section{MARCO LEGAL}

La discapacidad es una restricción, limitación o ausencia de la capacidad de las personas de realizar una actividad que, de acuerdo con la forma definida en el ámbito físico o socio-cultural, es considerada necesaria o parte de su desempeño y se debe diferenciar del término minusvalía que hace referencia a las dificultades que limitan 0 impiden el desempeño de una persona a causa de los obstáculos del entorno en el que se desenvuelve. (Ochoa y Leyva, 2008)

Esta nueva definición resulta del enfoque actual de los organismos internacionales, como la Organización de Naciones Unidas ONU y la Organización Mundial de la Salud OMS, en el que se abandonan visiones tradicionales de la discapacidad, que se ha tratado como un problema de salud manejado de manera aislada con miras a la rehabilitación y la ayuda de recursos humanos especializados. 
En estos últimos años se ha estado trabajando para que la discapacidad sea vista como un problema de derechos humanos, firmando protocolos y convenciones patrocinados por la ONU, en el que se da énfasis en la persona y no tanto en su discapacidad, y en la relación de la persona con su entorno físico, de manera que sea incluida en los sistemas normales de educación, trabajo y salud. Esto resulta en que la persona con discapacidad ahora sea un actor, un sujeto que forja su propio desarrollo.

El acceso a las edificaciones es un derecho contemplado tanto en el ámbito nacional como en el internacional. A partir de 1980, se crearon varias leyes y políticas destinadas a asegurar los derechos de las personas con discapacidad, abarcando temas de salud, rehabilitación, educación y empleo. En lo que se refiere a la accesibilidad arquitectónica los primeros documentos los encontramos hasta 1999.

A continuación se enumeran, en orden de publicación, los documentos relacionados con la accesibilidad universal:

1. Normas arquitectónicas para facilitar el acceso, tránsito y permanencia de personas con discapacidad y de la tercera edad a los establecimientos nacionales de la atención médica, al interior de la Secretaría de Salud y sus anexos.

Consiste en un manual desarrollado por la Secretaría de Estado en el Despacho de Salud, con número de Acuerdo 0862 del 07 de abril de 1999. Está dividido en tres secciones: las disposiciones generales, los requisitos arquitectónicos generales y los requisitos arquitectónicos específicos.

2. Política nacional para la prevención de la discapacidad, atención y rehabilitación integral de las personas con discapacidad, y la promoción y protección de sus derechos y deberes.

Esta política fue publicada en mayo 2004, sin embargo se venía desarrollando desde noviembre de 1999 y es a partir del 2001 que se logra integrar sus aspectos más importantes a la Estrategia de Reducción de la Pobreza.

3. Ley de equidad y desarrollo integral para las personas con discapacidad

Consta de 84 artículos, publicada en la Gaceta \# 30,832 del 25 de octubre 2005 bajo el Decreto \# 160-2005. Define discapacidad como cualquier tipo de deficiencia física, mental o sensorial, que en relación a la edad y medio social, Debido a que uno de los objetivos de esta política es asegurar el acceso de las personas 
con discapacidad al entorno físico, se desarrolla una línea estratégica de acción que involucra la participación del sector público y privado, para alcanzar medidas y acciones tendientes a eliminar las barreras urbanísticas y arquitectónicas, con el fin de mejorar la calidad de vida de las personas con discapacidad y de la sociedad en general. El objetivo de esta política es ejecutar programas que permitan incorporar las normas, requisitos y especificaciones técnicas, que consideren la accesibilidad en obras de infraestructura en general.

Encontramos datos específicos sobre accesibilidad en los centros educativos y que estos deben cumplir con las exigencias físicas para atender a los estudiantes con necesidades educativas especiales. Para asegurar y facilitar el acceso de las personas con discapacidad, las construcciones nuevas, ampliaciones, remodelaciones de edificios, parques, aceras, áreas verdes, jardines, plazas, vías públicas, servicios sanitarios u otros espacios que impliquen concurrencia de cualquier tipo y brinden atención al público, deberán construirse de acuerdo a las especificaciones técnicas que serán emitidas y reglamentadas por la Dirección General de Desarrollo para Personas con Discapacidad (DIGEDEPDI). Las instituciones públicas y privadas existentes, deben adecuar sus edificaciones para asegurar la accesibilidad, en un plazo máximo de tres 3 años a partir de la vigencia de esta Ley, o sea, a partir del 2008.

4. Convención sobre los derechos de las personas con discapacidad

Esta reunión efectuada en diciembre de 2006 bajo el marco de la 61 Asamblea, Convención sobre los derechos de las personas con discapacidad y el Protocolo Facultativo de la Convención, firmado por Honduras en marzo de 2007, busca corregir las injusticias que prevalecen en esta esfera. Entró en vigor en mayo de 2008 y actualmente 158 de 192 países ya se adhirieron al Convenio.

El documento se compone de un preámbulo y 50 artículos, que recogen derechos de carácter civil, político, social, económico y cultural. Resaltamos el Artículo 24, numeral 5 , que trata sobre el derecho a la Educación Superior para las personas con discapacidad.

5. Normas de actualización de la zonificación y normas de fraccionamiento, obras y uso de suelo en el Distrito Central

Estas normas se encuentran vigentes desde el 4 de abril del 2008 y en ellas encontramos 17 artículos referentes a la accesibilidad, características y dimensiones para accesos, circulaciones horizontales y verticales (rampas y escale- 
ras), rutas de evacuación y su señalización y especificaciones por tipologías especiales como auditorios, teatros, cines, salas de concierto, comedores, restaurantes, atención al público, instalaciones deportivas y almacenes.

\section{METODOLOGÍA}

\section{Enfoque}

En esta investigación, el problema de la accesibilidad universal se enfoca en el aspecto cualitativo. Se realizaron encuestas para saber la percepción del estudiante universitario respecto a la accesibilidad y se proyectaron propuestas arquitectónicas para solventar esos problemas.

\section{Temporalidad}

El estudio es de orden transversal pues se revela la situación actual del campus de la UNAH, en el aspecto físico.

\section{Población y muestra}

Se utilizó el $100 \%$ del universo. Se tomó en consideración el acceso a la primera planta de los 33 edificios que conforman la ciudad universitaria, los 28 estacionamientos, los 9 cruces peatonales y las 70 rampas de circulación entre los edificios.

El trabajo se realizó en varias etapas: primero, la recopilación de toda la información pertinente, luego, entrevista con los actores principales, posteriormente, se determinó el formato de los planos y finalmente, se procedió a la verificación de la accesibilidad en todos los elementos a ser estudiados.

Se cotejó la recopilación de toda la información obtenida con los distintos requerimientos arquitectónicos y la normativa legal de respaldo para validar la investigación. Se eligieron las instituciones reconocidas que por su naturaleza manejan manuales de accesibilidad concernientes a la población con discapacidad y a la calidad del espacio urbano. 


\section{RESULTADOS}

Las inversiones en el espacio universitario vienen creciendo a un ritmo esperanzador, solo el año pasado se invirtieron alrededor de 800 millones de lempiras y en el 2016, las inversiones rondarán los 600 millones de lempiras. Estas inversiones en el campus generan amplios beneficios porque provocan un efecto dominó en la economía. Mejoran las condiciones de empleo, aumentan la compra de materiales de construcción y colocan circulante en el mercado, además del beneficio social que esto provoca, que es la mejora de la calidad del espacio físico y la calidad estudiantil.

El retorno de estas mejoras tiene aún un impacto mayor que otros rubros porque cada lempira invertido en accesibilidad representa aumentar considerablemente el número de usuarios en contacto con la cultura. Sin embargo, lo más importante es saber que son ejecutadas y supervisadas por empresas hondureñas.

Las obras proyectadas mejorarán la capacidad instalada, este año se espera que aproximadamente 50,000 estudiantes pasen por las instalaciones de la CU. Se licitó la recuperación del Edificio C3, abandonado desde el año 2000, también se sometió a concurso la construcción del Edificio 1847 y se espera que este año comiencen las obras. Se están diseñando cuatro edificaciones más que superarán la inversión de los años anteriores. Debido a este gran salto en el crecimiento del espacio urbano, tomamos los elementos que componen el paisaje: edificios, estacionamientos, rampas y cruces peatonales, analizamos su accesibilidad y damos propuestas para que, a través de pequeñas obras, mejore la circulación de todas las personas que visitan el lugar.

Para mejor comprensión del trabajo, se dividió la CU en 7 sectores. En el Sector I, que consta de 7 edificios, están las 5 construcciones más antiguas, es también el "centro urbano" del complejo universitario. En el Sector II, se encuentran 4 edificios. En el Sector III, se contabilizaron 5 edificaciones, en el Sector IV se analizó el Palacio de los Deportes, el Sector V encontramos 5 construcciones, en el Sector VI también se analizaron 5 edificios y en el Sector VII se contabilizaron 6 edificaciones, haciendo un total de 33 construcciones. 
Figura 1. División de la UNAH en sectores. Plan de Accesibilidad Universal.

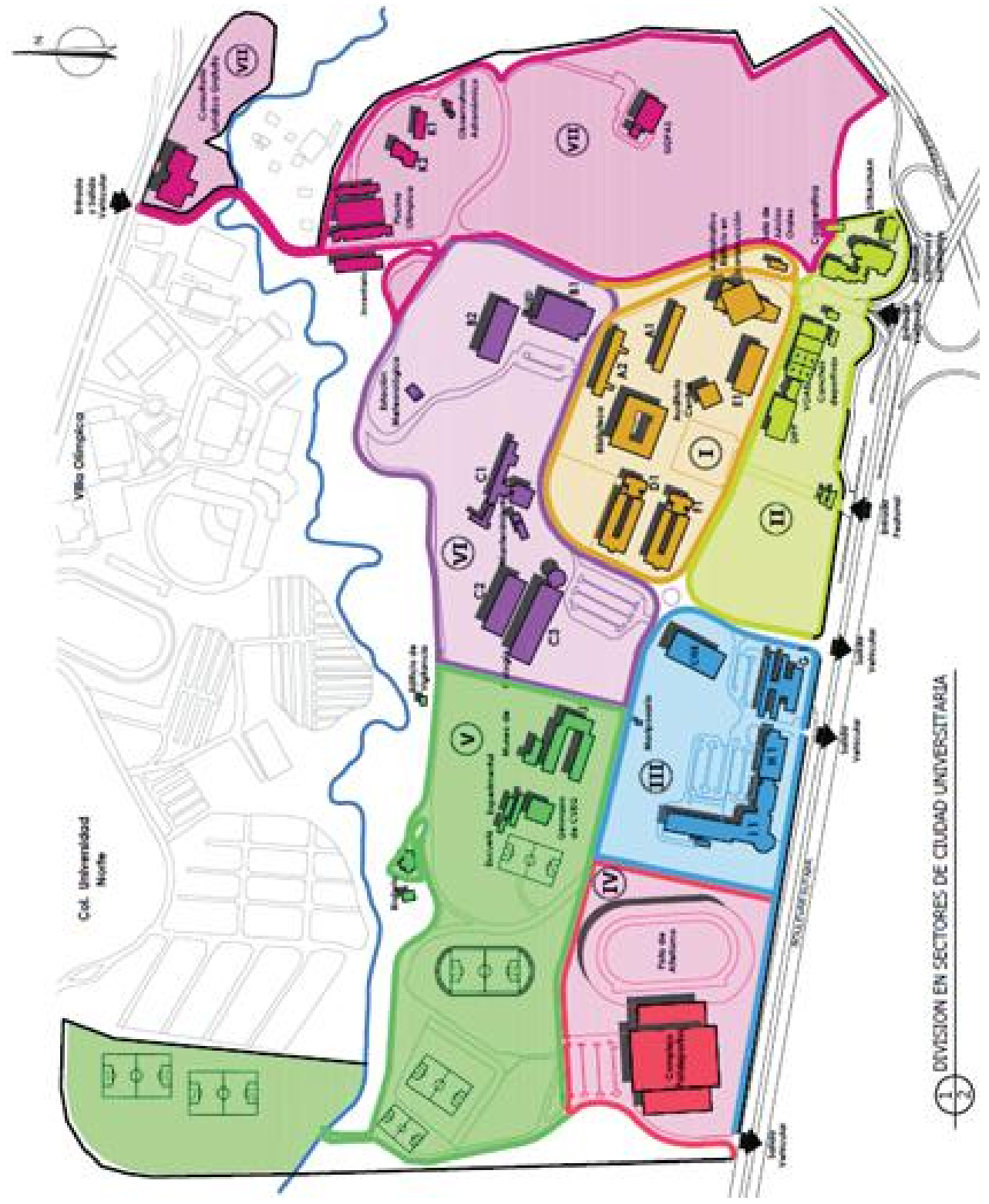

Fuente: Elaboración propia 


\section{Edificios}

Se realizó la verificación de la accesibilidad en un total de 33 edificaciones que componen el campus. No se tomó en consideración el nuevo edificio administrativo por encontrarse en la etapa final de su construcción. El análisis consistió en verificar la accesibilidad del estacionamiento más cercano y su acceso a la primera planta.

Los resultados obtenidos indican que de las 33 edificaciones solamente 13 de ellas son accesibles. Entre las edificaciones que no son accesibles, se necesitan desde pequeñas obras hasta adaptaciones complejas para tornar el campus $100 \%$ accesible. El $82 \%$ de las edificaciones tiene varios niveles, sin embargo, solamente 2 edificios cuentan con elevador. Un dato adicional es que ninguno tiene señalización en sistema Braille, aunque esto no es exigido en el reglamento de Metroplan.

Se encontró que existen edificios que dan atención al público, sin embargo, ninguno cuenta con módulos exclusivos para personas con discapacidad, a excepción del banco que está instalado en la primera planta del edificio administrativo. Se llama la atención respecto a que en el Auditorio Juan Lindo, edificación emblemática del campus, no existen espacios destinados a la permanencia de personas con discapacidad.

Figura 2. Acceso a la primera planta de los 33 Edificios que componen la Ciudad Universitaria. 2015

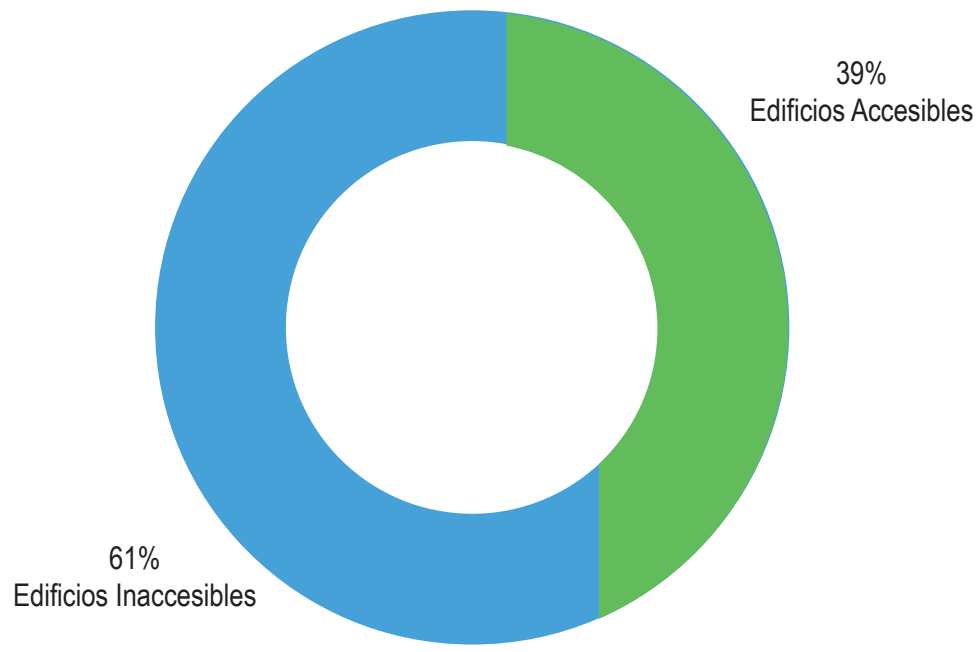

Fuente: Elaboración propia 


\section{Estacionamientos}

Se tomó en consideración los tres tipos de estacionamiento que funcionan en la ciudad universitaria, según su textura: los estacionamientos pavimentados, los estacionamientos de tierra y el estacionamiento a ambos lados de la calle pavimentada.

Existen 28 parques de estacionamiento, siendo 17 pavimentados y 11 de tierra. Hay capacidad para 1,149 plazas en los estacionamientos pavimentados y 619 plazas en los estacionamientos de tierra, sumando 1,768 en total. En la calle pavimentada se contabilizaron 474 vehículos estacionados, no se tomaron en cuenta los estacionamientos en las calles de tierra por ser un número variable, no existiendo una división definida de las plazas. Tenemos entonces que se pueden estacionar en el campus, alrededor de 2,242 vehículos. Ninguna de las plazas está reservada para personas con discapacidad. Adicionalmente, se verificó que aproximadamente 1,000 motos se estacionan en la hora pico, entre 6:00 y 7:00 pm., no existiendo lugares definidos para este tipo de vehículos

El transporte urbano, taxi y buses, no utilizan el interior de las instalaciones universitarias, porque permanecen fuera del límite del terreno de la UNAH, sin embargo, observamos también la accesibilidad entre la terminal y la entrada peatonal, que se encuentra descrita más adelante.

De acuerdo con el Decreto Ley 160-2005, por cada 20 plazas de estacionamiento debe haber 1 plaza para discapacitados, o sea, que deben existir al menos 30 plazas para discapacitados distribuidos en el campus. Las plazas deben tener una franja de $1.20 \mathrm{~m}$ para poder bajarse o subirse del carro con una silla de ruedas, conforme la siguiente figura, con el objeto de poder acceder al edificio.

No es útil solamente pintar un espacio de estacionamiento con el símbolo de accesibilidad si no hay como poder maniobrar con la silla de ruedas. Por tanto, lo ideal es que sean proyectados en número par, así la franja de circulación puede ser usado por 2 vehículos, dependiendo si la persona va manejando o es pasajero. Una barrera arquitectónica impenetrable son los topes de la llanta en los estacionamientos, los cuales tornan aún más difícil entrar en los distintos edificios. 
Figura 3. Calles y estacionamientos en CU. Plan de Accesibilidad Universal

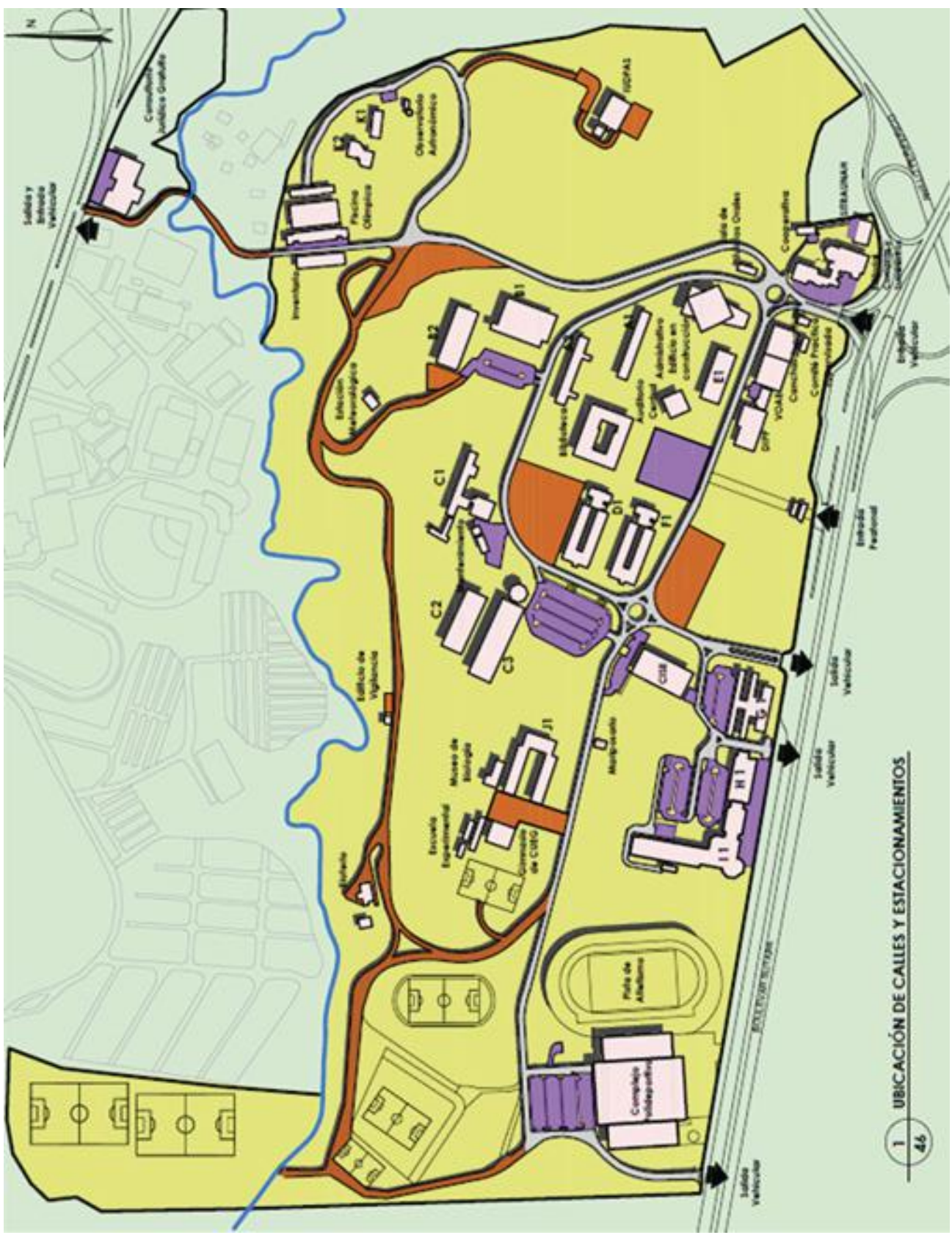

Fuente: Elaboración propia 
Figura 4. Esquema de utilización de la franja para maniobras en las plazas para discapacitados

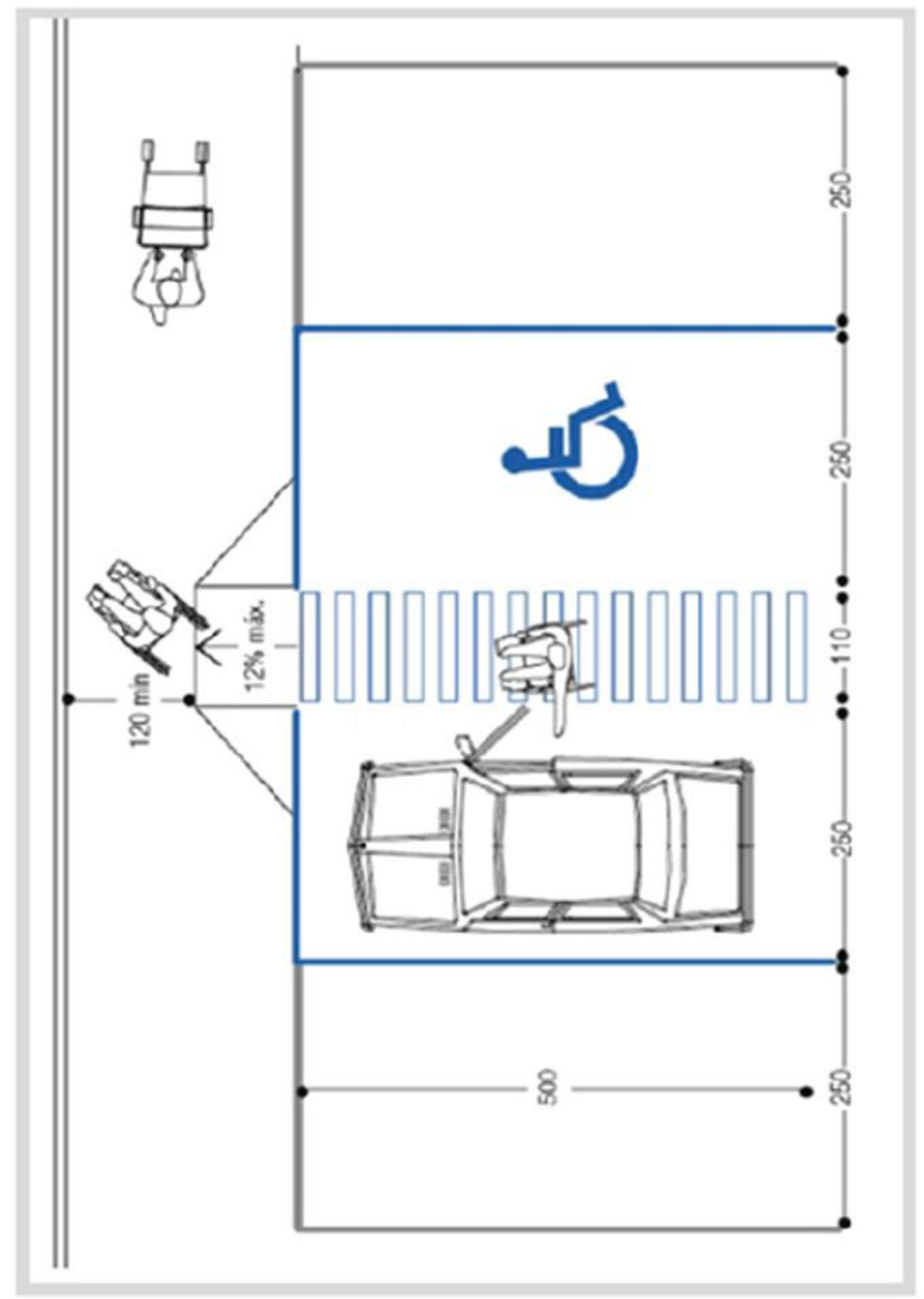

Fuente: Manual para Alcanzar la Inclusión en el Aula Universitaria. 2012 Fundación ONCE. Universidad Politécnica de Cataluña. España. 


\section{Rampas}

Se contabilizaron 70 rampas de circulación entre los edificios en todo el campus. Se analizaron por sector y se dividieron por la inclinación que presenta cada una. Es decir, se asignó el color verde en los planos para aquellas que presentan una inclinación máxima de $10 \%$, el color amarillo para las rampas que tienen una inclinación entre $10 \%$ y $12 \%$, finalmente se pintaron de rojo las rampas cuya inclinación es superior a los $12 \%$.

Las características encontradas en las rampas de circulación entre los edificios se resumen a que el $100 \%$ cumple con el ancho mínimo establecido de $1.20 \mathrm{~m}$ y el $59 \%$ no tiene la pendiente adecuada. Las rampas tienen una longitud mayor a $5.00 \mathrm{~m}$ y ninguna cuenta con descansos intermediarios. Todas poseen un acabado de piso adecuado pero solo el $12 \%$ tiene barandal bilateral.

Se verificó la pendiente de cada una de las rampas y el resultado fue el siguiente:

- 31 rampas están dentro de los parámetros considerados accesibles o autónomos, quiere decir, que la persona puede salvar las rampas sin la ayuda de otra persona.

- 24 rampas poseen una inclinación entre 10\% y 12\%, haciendo que sea necesaria la intervención de una persona para ayudar a circular por la ruta.

- 15 rampas tienen arriba del $12 \%$ de inclinación, haciendo casi imposible poder circular en ellas, mismo con la ayuda externa, son rampas muy inclinadas que ponen la seguridad en riesgo, siendo plausible un accidente con consecuencias graves.

Figura 5. Inclinación, en porcentaje, de las rampas de circulación entre los edificios de $\mathrm{CU}$

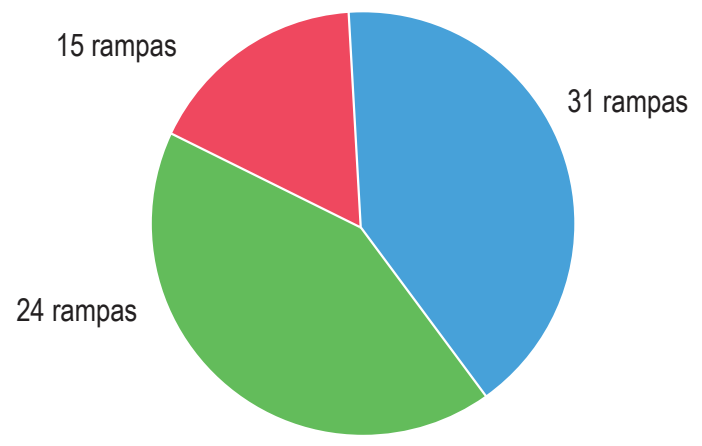




\section{Cruces peatonales}

Se contabilizaron 18 cruces peatonales en toda la CU, los cuales están definidos y marcados. No han recibido el mantenimiento adecuado, sin embargo, se nota el esfuerzo de las autoridades por mantenerlos visibles y libres de obstáculos.

Figura 6. Cruces peatonales en CU. Plan de Accesibilidad Universal.

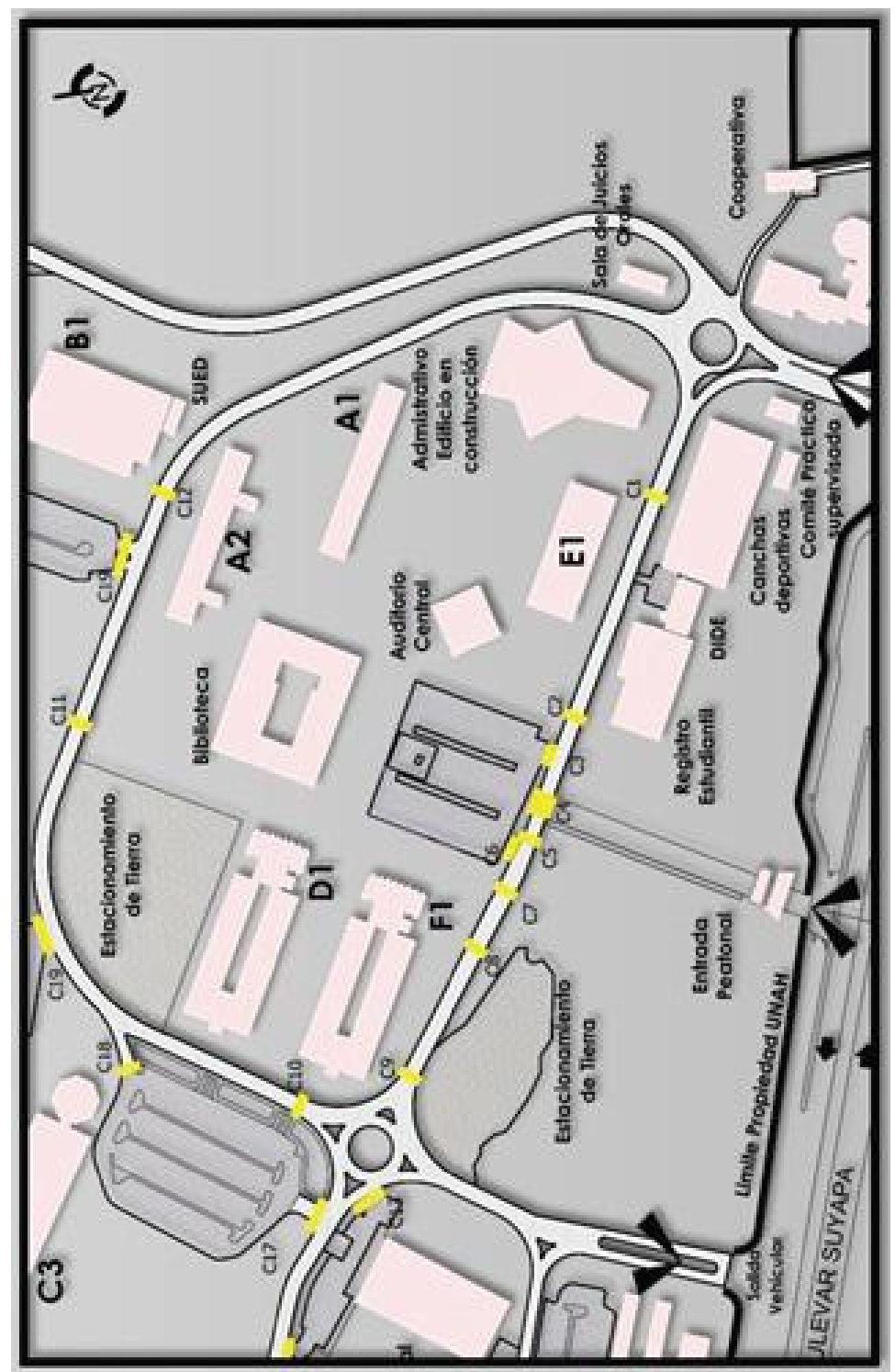

Fuente: Elaboración propia 
La totalidad de las circulaciones exteriores analizadas tiene un acabado adecuado, pero la mayoría no cuenta con cambios de textura en los cruces y descansos, aspecto que es importante para la orientación de las personas invidentes. Sin embargo, la mayoría cuenta con un ancho adecuado y constante.

Figura 7. Cruce entrada peatonal. Nótese la grada provocada por el bordillo, la textura irregular del piso, la señalización casi desapareciendo y los obstáculos para evitar que los carros se estacionen.

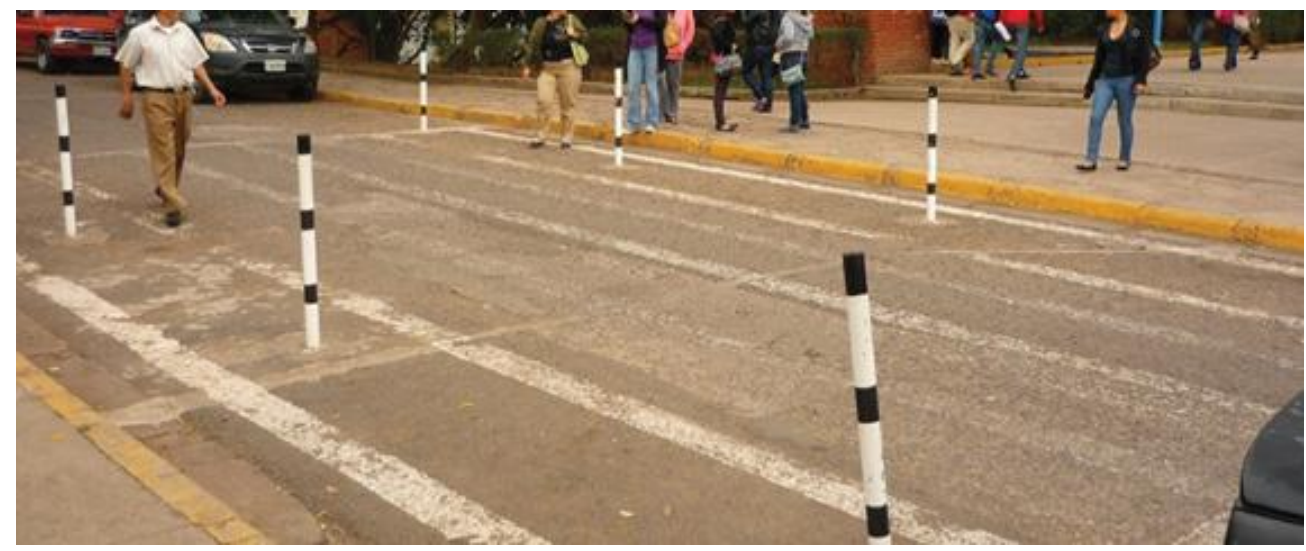

\section{CONCLUSIONES}

El papel que desempeña el entorno físico, para facilitar la participación de las personas con discapacidad, es el punto crucial en este trabajo. El Informe aporta pruebas sustanciales de los obstáculos arquitectónicos a que se enfrentan todas las personas. Además, las normas básicas de urbanidad tampoco merecen el respeto por parte de toda la población universitaria. Los servicios básicos para las personas con discapacidad en la CU son particularmente muy sensibles a las deficiencias que presentan tanto en asistencia como en apoyo.

La verificación realizada sobre el cumplimiento de las normas de accesibilidad en las edificaciones, estacionamientos, rampas y cruces peatonales concluye que las mismas no están adaptadas con un diseño universal, es decir que, en su mayoría no son accesibles; y de las que se pudo observar que sí eran accesibles, por lo general no eran funcionales. 
Falta accesibilidad en dos tercios de los edificios de la universidad. Esto desalienta a las personas con discapacidad y les impide acceder a una educación de calidad. A pesar de contar con leyes sobre la accesibilidad, aunque tengan una antigüedad de 15 años, estas tienen un bajo nivel de cumplimiento, mismo en la Universidad Nacional Autónoma de Honduras.

\section{BIBLIOGRAFÍA}

Asociación Nacional de Universidades e Instituciones de Educación Superior de México (2004) Manual para la Integración de las Personas con Discapacidad en las Instituciones de Educación Superior. México.

Fundación ONCE (2012) Manual para Alcanzar la Inclusión en el Aula Universitaria.

Observatorio Universidad y Discapacidad. Universidad Politécnica de Cataluña.

España.

Instituto Nacional de Estadísticas. INE (2013) XVII Censo de Población y VI de Vivienda. Honduras.

Ley de Equidad y Desarrollo Integral para las Personas con Discapacidad. Decreto 160 - 2005. Honduras.

North Carolina State University (1997) Los Principios del Diseño Universal. Centro para el Diseño Universal. USA.

Organización de las Naciones Unidas. ONU. (2006) Convención sobre los Derechos de las Personas con Discapacidad. New York, EUA.

Organización Mundial de Salud, OMS y Banco Mundial. (2001) Informe Mundial sobre la Discapacidad.

Organización de las Naciones Unidas ONU (1993) Normas Uniformes sobre Igualdad de Oportunidades para Personas con Discapacidad. New York, EUA.

Secretaría del Interior y Población (2010) Plan Nacional de Accesibilidad Universal. Dirección General de Desarrollo para las Personas con Discapacidad DIGEDEPDI. Honduras.

Solís, R. (2009) Revisión de la Accesibilidad de dos Edificios de un Campus Universitario. Anuario 2009 Administración y Tecnología para el Diseño. Mérida, México.

Ochoa, D. García, M. Leiva, S. (2008) Verificación de accesibilidad arquitectónica en edificios públicos según reglamento de construcción vigente en Tegucigalpa. Trabajo Final. Asignatura: Seminario de Investigación. Escuela de Arquitectura y Diseño. Universidad Nacional Autónoma de Honduras. 